\title{
Nonlinear shallow water tropical instability waves on the equatorial $\beta$-plane: Genesis of two distinct types of waves
}

\author{
C. Zhou ${ }^{1}$ and J. P. Boyd ${ }^{1}$ \\ Received 14 August 2009; revised 23 October 2009; accepted 29 October 2009; published 8 December 2009.
}

[1] The nonlinearity of Tropical Instability Waves (TIWs) is studied using shallow-water equations with various mean states from the equatorial Pacific Ocean. In the early linear stage, unstable TIWs, centered near $5^{\circ} \mathrm{N}$ with a wavelength about $1000 \mathrm{~km}$ and a period about one month, dominate the whole domain. However neutral Yanai waves with periods about 15-22 days emerge near the equator when the unstable TIWs grow into fully nonlinear vortices and begin to rotate, which stabilizes the mean states substantially. The strength of these Yanai waves are sensitive to the instability of the initial mean flow. Meanwhile the TIWs centered near $5^{\circ} \mathrm{N}$ are slowed down and weakened. The external forcing terms are found to be important for them to retain their dominance from $3^{\circ} \mathrm{N}$ to $7^{\circ} \mathrm{N}$ and also be able to suppress the late emerging Yanai waves if strong enough. Citation: Zhou, C., and J. P. Boyd (2009), Nonlinear shallow water tropical instability waves on the equatorial $\beta$-plane: Genesis of two distinct types of waves, Geophys. Res. Lett., 36, L23605, doi:10.1029/2009GL040499.

\section{Introduction}

[2] Tropical instability waves (TIWs) are westward propagating oscillations observed in both equatorial Pacific and Atlantic oceans. They were first detected in satellite infrared images as cusplike deformations of the North Equatorial Front (NEF) in the Pacific ocean by Legeckis [1977]. Subsequent measurements observed TIWs with various wavelengths and periods, in ranges of $600-2000 \mathrm{~km}$ and 16-40 days, respectively [Qiao and Weisberg, 1995]. These TIWs generally fall into two categories. The first has periods around 15-23 days, is most prominent in meridional velocity, and has been observed within a few degree of the equator [Halpern et al., 1988; Qiao and Weisberg, 1995; Flament et al., 1996; Kennan and Flament, 2000; Lyman et al., 2007]. The second has periods around one month and has been observed in sea surface height, thermocline depth, velocity, and subsurface temperature centered about $5^{\circ} \mathrm{N}$ [Miller et al., 1985; Flament et al., 1996; Kennan and Flament, 2000; Lyman et al., 2005, 2007; Shinoda et al., 2008]. Flament et al. [1996] and Kennan and Flament [2000] observed two drastically different propagation speeds of TIWs at the same time, $0.8 \mathrm{~m} \mathrm{~s}^{-1}$ along the equator and $0.3 \mathrm{~m} \mathrm{~s}^{-1}$ along $4.5^{\circ} \mathrm{N}$. They suggested they were two distinct phenomena. Lyman et al. [2007] also observed two different types of TIWs: unstable Rossby waves at a period of about 33 days characterized by subsurface temperature at $5^{\circ} \mathrm{N}$ and Yanai waves at a period of about

\footnotetext{
${ }^{1}$ Department of Atmospheric, Oceanic and Space Sciences, University of Michigan, Ann Arbor, Michigan, USA.
}

Copyright 2009 by the American Geophysical Union. 0094-8276/09/2009GL040499
17 days characterized by fluctuations in meridional velocity at the equator and in subsurface temperature at $2^{\circ} \mathrm{N}$ and $2^{\circ} \mathrm{S}$.

[3] Nonlinear models by McCreary and $Y u$ [1992] and $Y u$ et al. [1995] produced two waves with distinct periods (wave 1 with periods about 20 days and wave 2 with periods about 40-50 days [Yu et al., 1995]). Close look indicates these two waves have almost identical phase speeds, which suggests they may arise from the same critical latitude [Proehl, 1996]. The nonlinear 2-1/2-layer model by Donohue and Wimbush [1998] also generated a 15-day wave with a phase speed about $0.88 \mathrm{~m} \mathrm{~s}^{-1}$ and a strong meridional signal centered on the equator and a 30-day wave with a phase speed about $0.42 \mathrm{~m} \mathrm{~s}^{-1}$ and sea level maxima near $6^{\circ} \mathrm{N}$. They argued that the 15-day wave arose from the barotropic instability of the cyclonic shear of south flank of the north South Equatorial Current (SEC) while the 30-day waves arose from both the barotropic instability of the anticyclonic shear of the north flank of SEC and the baroclinic conversion near the core of SEC. With observational data and a projected linearized model, Lyman et al. [2007] were able to identify the 33-day waves as unstable first meridional mode Rossby waves; however, the fast 17-day waves did not show up as unstable waves when the authors ran the linearized model with five vertical modes and gradually increased the shear of the mean equatorial currents. So how these 17-20 day Yanai-like waves with relative fast propagating speed are generated still remains unclear.

[4] In this study, we focus on the TIWs arising from the barotropic instability and investigate how the nonlinearity changes their strength, periods, etc. Especially, we explain how the two distinct types of waves with different propagating speeds arise. We then evaluate the effect of external forcings on these two types of waves.

\section{Model}

[5] The nonlinear shallow-water equations over a flat bottom on equatorial beta plane are

$$
\begin{aligned}
& u_{t}+u u_{x}+v u_{y}-y \beta v+g h_{x}=0 \\
& v_{t}+u v_{x}+v v_{y}+y \beta u+g h_{y}=0 \\
& h_{t}+u h_{x}+v h_{y}+h\left(u_{x}+v_{y}\right)=0
\end{aligned}
$$

where $u$ and $v$ are the eastward and northward velocities, $h$ is the total depth, $g$ is the gravitational constant, and $\beta$ is Rossby parameter at the equator. This is also called the 'oneand-a-half-layer' model because it describes two-layer fluid in the hydrostatic approximation when the lower layer is 

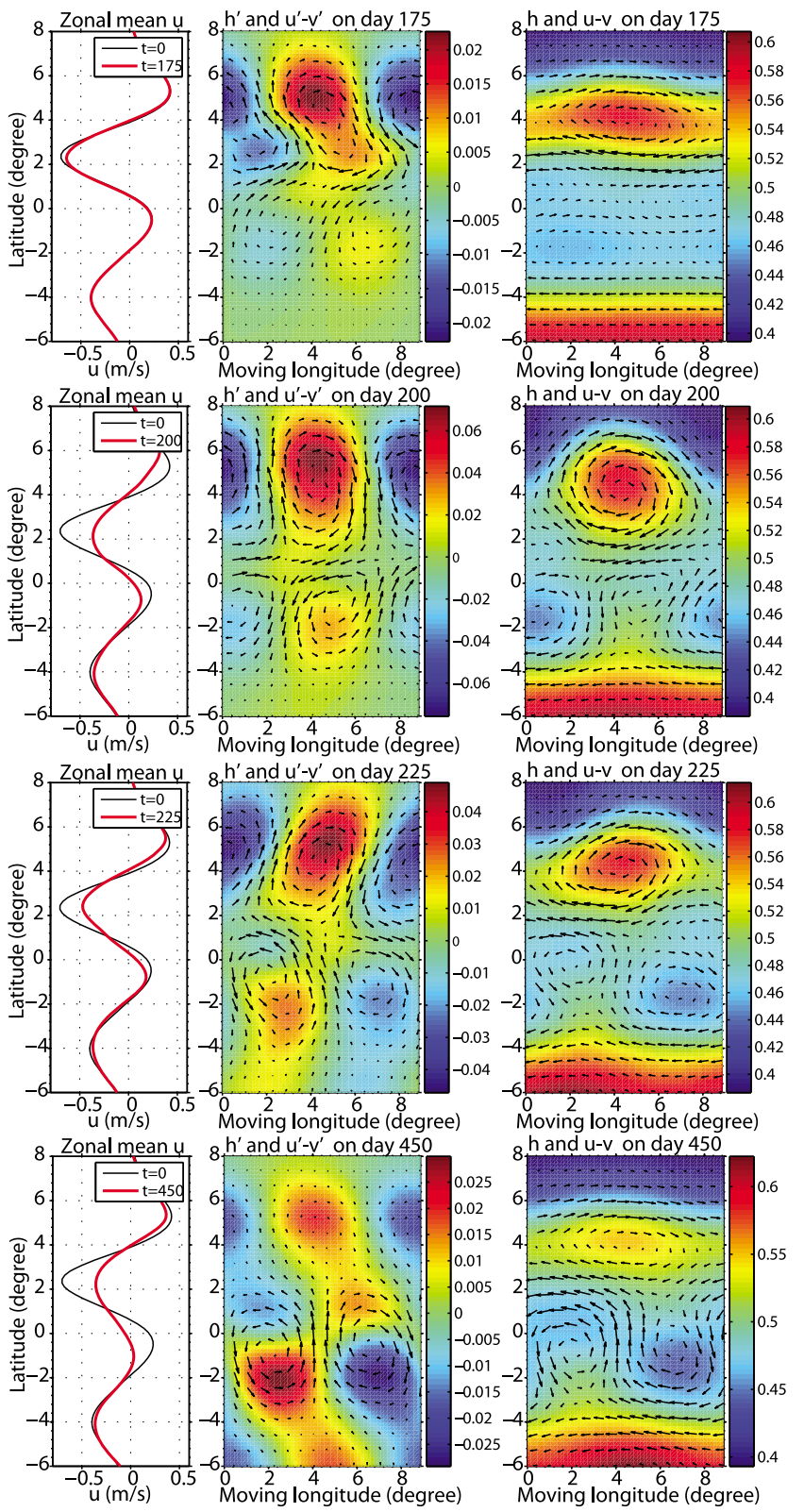

Figure 1. (left) Zonal mean velocity, (middle) zonal deviation $h^{\prime}$ and $u^{\prime}-v^{\prime}$, and (right) $h$ and $u-v$ on a moving coordinate following a positive $h^{\prime}$ center near $5^{\circ} \mathrm{N}$ on day $175,200,225$, and 450 .

infinitely deep. In this study results are not sensitive to a realistic range of $h$ in the equatorial ocean from $0.2 \mathrm{~m}$ to $0.8 \mathrm{~m}$. A value of $0.5 \mathrm{~m}$, approximately equal to the equivalent depth of the 1st baroclinic mode, is used. Numerical schemes follow Boyd [1998]. The spatial derivatives in both latitude and longitude are approximated by centered eighth-order differences. For the temporal derivatives, we use the 4 th order Runge-Kutta for the first two steps since it is self starting and the 3rd order Adams-Bashforth after. 6th order dissipation is used with a hyperviscosity coefficient chosen in a way such that the $e$-folding time of the shortest $2 \Delta x$ wave is 0.1 day. The domain size is $\left[20^{\circ} \mathrm{S}, 20^{\circ} \mathrm{N}\right]$ in latitude, and $300^{\circ}$ in longitude. In latitude, due to the equatorial wave guide effect, there is no substantial wave activity on north and south boundaries. So we simply keep using the initial values on these boundaries during the integration. In longitude, we use heavy damping near the boundaries, so-called "sponge layers". The spatial resolution is $5 \mathrm{~km}$ and the time step is 4 minutes.

\section{Nonlinear Evolution}

\subsection{Free Nonlinear Evolution}

[6] The nonlinear evolution of TIWs is initialized with a small random perturbation to the initial mean state shown in Figure 1 (see also Animation S1 for the 450-day time evolution). ${ }^{1}$ This mean state is based on Hansen and Paul [1984, Figure 4] which was based on measurements made by 20 satellite-tracked surface drifters in the region $10^{\circ} \mathrm{S}-10^{\circ} \mathrm{N}$, $130-140^{\circ} \mathrm{W}$, from June 6 to October 27 in 1979. The evolution of the waves originating near the center of the $300^{\circ}$ longitude band is analyzed.

[7] Before day 175 , in the latitudinal range from $6^{\circ} \mathrm{S}$ to $8^{\circ} \mathrm{N}$, TIWs with a wavelength of $995 \mathrm{~km}$, a period of 29 days, growing exponentially with an $e$-folding time of 11.4 days, and similar wave pattern as that on day 175 in Figure 1 dominate the whole domain. These results are consistent with the stability analysis following Philander [1978]. As shown in Figure 1, after day 175, the TIWs rapidly grow into fully nonlinear vortices and further growth stops around day 200 . Three related things happen during this period. First, the vortex shown in total $h$ field begins to rotate, changing its oval shape on day 175 to disk shape on day 200 . Second, the rotation decouples the components of TIWs at different latitudes. The wave near the equator detaches from the wave near $5^{\circ} \mathrm{N}$ and propagates faster to the west. Third, the rotation also stabilizes the zonal mean substantially. Wavelengths of these waves do not change during the process. On day 225 , the vortex rotates back to an oval shape and the zonal mean is restored partially. However, this process is irreversible. The wave near the equator keeps moving faster to the west. And the wave near $5^{\circ} \mathrm{N}$ continue to surrender its energy to the fast wave near the equator after day 225 and reaches some quasiequilibrium after day 350 . On day 450 the fast wave near the equator has relative stronger amplitude than the wave near $5^{\circ} \mathrm{N}$.

[8] Figure 2 shows how $v$ along the equator and $5^{\circ} \mathrm{N}$ in a selected region near the center in longitude varies with time. Before day 175, the period is 29 days and the phase speed is $0.4 \mathrm{~m} \mathrm{~s}^{-1}$ at both latitudes; after day 200 , the period is 20 days and phase speed is $0.58 \mathrm{~m} \mathrm{~s}^{-1}$ at the equator, and the period is about 36 days and the phase speed is $0.31 \mathrm{~m} \mathrm{~s}^{-1}$ at $5^{\circ} \mathrm{N}$. Close look indicates that the 20 -day wave dominates $\left[4^{\circ} \mathrm{S}, 2^{\circ} \mathrm{N}\right]$ and the 36 -day wave dominates $\left[3^{\circ} \mathrm{N}, 8^{\circ} \mathrm{N}\right]$. Figure 2 (right) also shows that the 36-day wave keeps losing its energy to the 20-day wave till day 350 .

[9] The genesis of these two types of waves with distinct phase speeds could be explained through the linear dynamics. Recall that zonal wavenumber $k=1$ is a dimensional wavelength of $995 \mathrm{~km}$ while its second harmonic $(k=2)$ is a wavelength of $498 \mathrm{~km}$. Even though the TIWs around day 200 are fully nonlinear, Fourier analysis indicates the $k=$ 1 waves are still dominant, about 10 times larger than the second harmonics on day 200 at $2^{\circ} \mathrm{S}$ (center of the fast 20 -day

\footnotetext{
${ }^{1}$ Auxiliary materials are available in the HTML. doi:10.1029/ 2009GL040499.
} 

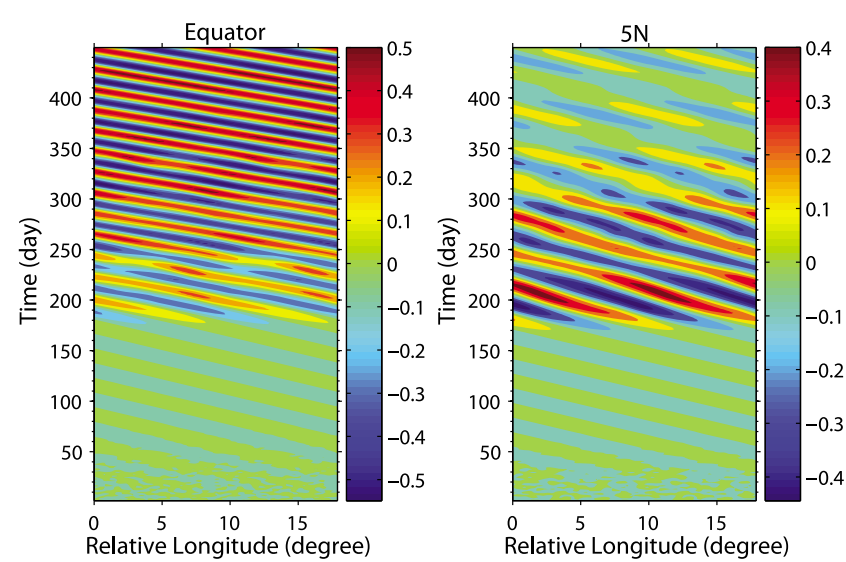

Figure 2. Time-longitude plots of $v$ along the equator and $5^{\circ} \mathrm{N}$. Before the most unstable wave reaches to its fully nonlinear stage around day 200, the periods are 29 days at both the equator and $5^{\circ} \mathrm{N}$. After day 250 , the periods are 20 days at the equator and about 36 days at $5^{\circ} \mathrm{N}$.

wave) and $5^{\circ} \mathrm{N}$ (center of the 36-day wave). The stabilized zonal mean on day 200 does not support the original 29-day unstable mode anymore. The whole large perturbation field must be decomposed to a new set of free modes existing with the new stabilized zonal mean. Since the zonal mean on day 200 closely resembles the zonal mean on day 450 , we use the latter as an example. As shown in Figure 3a, the only discrete neutral Rossby-like waves now is a 20-day Yanai wave and all other neutral Rossby-like waves are continuous. It also has a 41-day discrete unstable growing mode centered $5^{\circ} \mathrm{N}$ with an $e$-folding time of 36 days and several other slower growing discrete modes. As the zonal deviation of the fast wave from $4^{\circ} \mathrm{S}$ to $2^{\circ} \mathrm{N}$ on day 450 and the Yanai mode in Figure $3 \mathrm{~b}$ have the same wavelength, period and similar wave pattern, we identify this fast 20-day wave as the neutral Yanai wave. While the near 36 -day waves centered $5^{\circ} \mathrm{N}$ vary with time and involve both continuous neutral modes and discrete unstable modes which makes it impossible to identify them with any particular mode.

\subsection{Sensitivity of the Late Emerging Yanai Wave to the Zonal Mean}

[10] Although the initial zonal mean used in the previous subsection is representative of a very large collection of data in one year, substantial variations of strength and structure are known to occur from year to year. Here we just simply rescale the magnitude of the zonal mean to a wider range to represent such interannual variations.

[11] The magnitude $U_{\max }$ of our "typical" zonal mean is $0.7 \mathrm{~m} \mathrm{~s}^{-1}$. If we rescale $U_{\max }$ up to $1.0 \mathrm{~m} \mathrm{~s}^{-1}$ (decreasing the $e$-folding time of the most unstable mode from 11.4 days to 7.3 days), then the late emerging 15-day Yanai wave will dominate from $6^{\circ} \mathrm{S}$ to $6^{\circ} \mathrm{N}$ as shown in Figure 4 (middle). The initial 25-day TIW near $5^{\circ} \mathrm{N}$ do not have any remnant near $5^{\circ} \mathrm{N}$. Figure 4 (left) also shows that the structure of the zonal mean on day 450 without forcing terms differs from the initial structure significantly. However, if we rescale $U_{\max }$ down to $0.5 \mathrm{~m} \mathrm{~s}^{-1}$ (increasing the $e$-folding time of the most unstable mode from 11.4 days to 18.3 days), then the late emerging Yanai wave with a period of 22 days will have such weak strength that the period can only be obtained from the Fourier
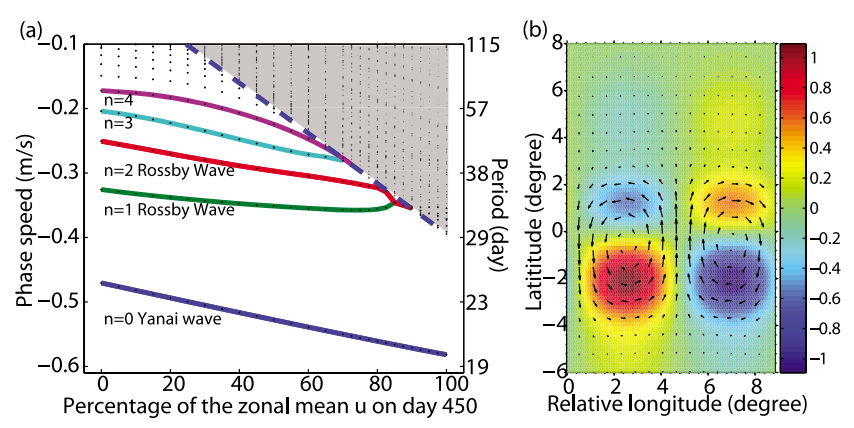

Figure 3. (a) Phase speed and periods of the free modes with a wavelength of $995 \mathrm{~km}$ when increasing the background zonal mean from 0 to $100 \%$ of the zonal mean on day 450. Blue dashed line denotes where the phase speed equals the minimum value of the zonal mean. Neutral modes in the shaded area are continuous. (b) The free Yanai wave with a wavelength of $995 \mathrm{~km}$ wave existing with $100 \%$ of the zonal mean on day 450 .

analysis (figures are not shown here). The initial 32-day wave with modified wave structure near the equator still dominates from $6^{\circ} \mathrm{S}$ to $8^{\circ} \mathrm{N}$.

\subsection{Forced Nonlinear Evolution}

[12] The sensitivity of the strength of the late emerging Yanai wave is largely due to the lack of external forcing terms in our nonlinear shallow-water model. Here we use Newtonian relaxation to represent the external forces, like wind stresses, by adding nudging terms $(\bar{u}-u) / \tau,(\bar{v}-v) / \tau$ and $(\bar{h}-h) / \tau$ to the right hand sides of equations (1), (2) and (3) respectively. They act to nudge the flow back towards the initial mean state $\bar{u}, \bar{v}(=0)$ and $\bar{h}$ with a relaxation time of $\tau$.

[13] Figure 4 (right) shows the zonal deviation $h^{\prime}, u^{\prime}-v^{\prime}$ on day 450 with a relaxation time of 60 days for an increased $U_{\max }$ of $1.0 \mathrm{~m} \mathrm{~s}^{-1}$. Comparing with the result without nudging terms on day 450 shown in Figure 4 (middle), the wave near $5^{\circ} \mathrm{N}$ now has large amplitude and dominates from $3^{\circ} \mathrm{N}$ to $8^{\circ} \mathrm{N}$. The Yanai wave is restricted to $4^{\circ} \mathrm{S}$ to $2^{\circ} \mathrm{N}$. The stabilized zonal mean is also nudged close to the initial mean as shown in Figure 4 (left).

[14] Table 1 gives the periods of TIWs centered about $5^{\circ} \mathrm{N}$ and late emerging Yanai waves near the equator for $U_{\max }=$ $0.7 \mathrm{~m} \mathrm{~s}^{-1}$ with various relaxation times, measured after the

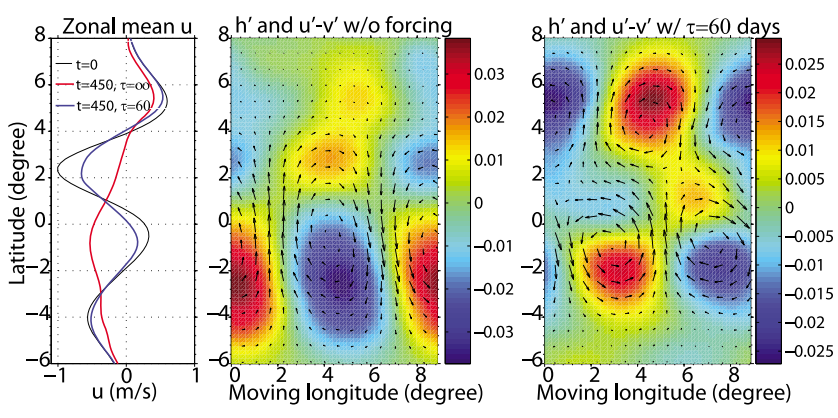

Figure 4. (left) Zonal mean velocity without nudging terms $(\tau=\infty)$ and with forcing terms $(\tau=60$ days $)$ for $U_{\max }=1.0 \mathrm{~m}$ $\mathrm{s}^{-1}$, (middle) zonal deviation $h^{\prime}$ and $u^{\prime}-v^{\prime}$ on day 450 without nudging terms, and (right) zonal deviation $h^{\prime}$ and $u^{\prime}-v^{\prime}$ on day 450 with nudging terms ( $\tau=60$ days). 
Table 1. Periods of TIWs Centered About $5^{\circ} \mathrm{N}$ and Late Emerging Yanai Waves Near the Equator for $U_{\max }=0.7 \mathrm{~m} \mathrm{~s}^{-1}$ After the Nonlinear Adjustment With Various Relaxation Times ${ }^{\mathrm{a}}$

\begin{tabular}{lcc}
\hline Relaxation Time $\tau$ & $5^{\circ} \mathrm{N}$ & Yanai \\
\hline w/o nudging & 36 & 20 \\
90 days & 34 & 21.5 \\
60 days & 33 & 22 \\
30 days & 32 & suppressed \\
\hline
\end{tabular}

${ }^{\mathrm{a}}$ The period of TIWs during the linear stage is 29 days over the domain.

nonlinear adjustment. Nudging terms tend to bring the periods of the waves near $5^{\circ} \mathrm{N}$ towards the initial period in the linear stage. For a relaxation time less than 30 days, the Yanai wave is suppressed. If we increase $U_{\max }$, then we need to decrease $\tau$ to suppress the late emerging Yanai waves.

\section{Summary and Discussion}

[15] In this study, we investigated how the nonlinearity could change the behavior of the TIWs and provide a new mechanism to explain the coexistence two different types of TIWs. The neutral Yanai waves are shown to emerge as the initial TIWs grow into full nonlinear vortices and stabilize the zonal mean significantly. The strength and structure of these Yanai waves are highly dependent on the instability of the initial mean states if no forcing terms are present. Such sensitivity could largely be eliminated by adding climatological nudging terms to the equations.

[16] One limitation of this study is the shallow-water model is only suitable for TIWs arising mainly from barotropic instability. For TIWs arising mainly from baroclinic or frontal instability, new models are needed to examine their nonlinear adjustment processes. However, we expect it is still possible for neutral Yanai modes to emerge if the nonlinear TIWs could stabilize the zonal mean significantly, regardless of whether the TIWs arise from barotropic or baroclinic instability.
[17] Acknowledgments. This work was supported by NSF grants OCE-0451951 and ATM-0723440.

\section{References}

Boyd, J. P. (1998), High order models for the nonlinear shallow water wave equations on the equatorial beta-plane with application to Kelvin wave frontogenesis, Dyn. Atmos. Oceans, 28, 69-91.

Donohue, K., and M. Wimbush (1998), Model results of flow instabilities in the tropical Pacific Ocean, J. Geophys. Res., 103, 21,401-21,412.

Flament, P., S. Kennan, R. Knox, P. Niiler, and R. Bernstein (1996), The three-dimensional structure of an upper ocean vortex in the tropical Pacific Ocean, Nature, 383, 610-613.

Halpern, D., R. Knox, and D. Luther (1988), Observations of 20-day period meridional current oscillations in the upper ocean along the Pacific equator, J. Phys. Oceanogr., 18, 1514-1534.

Hansen, D., and C. Paul (1984), Genesis and effects of long waves in the equatorial Pacific, J. Geophys. Res., 89, 431-440.

Kennan, S., and P. Flament (2000), Observations of a tropical instability vortex, J. Phys. Oceanogr., 30, 2277-2301.

Legeckis, R. (1977), Long waves in eastern equatorial Pacific Ocean: View from a geostationary satellite, Science, 197, 1179-1181.

Lyman, J., D. Chelton, R. deSzoeke, and R. Samelson (2005), Tropical instability waves as a resonance between equatorial Rossby waves, J. Phys. Oceanogr., 35, 232-254.

Lyman, J. M., G. C. Johnson, and W. S. Kessler (2007), Distinct 17-and 33-day tropical instability waves in subsurface observations, J. Phys. Oceanogr., 37 , 855-872, doi:10.1175/JPO3023.1.

McCreary, J., and Z. Yu (1992), Equatorial dynamics in a 2-1/2-layer model, Prog. Oceanogr., 29, 61-132.

Miller, L., D. Watts, and M. Wimbush (1985), Oscillations of dynamic topography in the eastern equatorial Pacific, J. Phys. Oceanogr., 15, $1759-1770$

Philander, S. G. H. (1978), Instabilities of zonal equatorial currents: 2, J. Geophys. Res., 83, 3679-3682.

Proehl, J. A. (1996), Linear stability of equatorial zonal flows, J. Phys. Oceanogr., 26, 601-621.

Qiao, L., and R. Weisberg (1995), Tropical instability wave kinematics: Observations from the tropical instability wave experiment, J. Geophys. Res., 100, 8677-8693.

Shinoda, T., G. N. Kiladis, and P. E. Roundy (2008), Statistical representation of equatorial waves and tropical instability waves in the Pacific Ocean, Atmos. Res., 94, 37-44.

Yu, Z., J. McCreary, and J. Proehl (1995), Meridional asymmetry and energetics of tropical instability waves, J. Phys. Oceanogr., 25, $2997-$ 3007 .

J. P. Boyd and C. Zhou, Department of Atmospheric, Oceanic and Space Sciences, University of Michigan, Ann Arbor, MI 48109-2143, USA. (zhouc@umich.edu) 